\title{
Priority Traffic Modeling in Multicellular Communication Networks
}

\author{
Pietro Camarda, Giovanna Schiraldi and Fabrizio Talucci \\ Dipartimento di Elettrotecnica ed Elettronica, Politecnico di Bari, Italy
}

In this paper we analyze a cellular communication environment which offers specific users priority access to network resources. We foresee a finite number of widespread disparate mobiles moving in a geographical region covered by a finite set of cells. Each user belongs to a class characterized by a specific service, load and mobility profile. In this paper we will consider two classes, namely high priority class and low priority class. The developed analytical network model, validated by simulation experiments, allows us to evaluate the main system performance parameters in terms of new call blocking probability, hand-off blocking probability and forced termination probability.

Keywords: cellular communication networks, priority traffic, analytical and simulation model.

\section{Introduction}

Constant growth of the number of subscribers and the large variety of services requested from cellular communication networks are driving a new trend in the telecommunications industry to provide ubiquitous access and support for users characterized by different service, load and mobility profiles. The system considered in this paper covers a geographical region by a finite number of cells. The model assumes a finite population of users moving in this finite set of cells. Each user communicates using a bidirectional link (up-link and down-link) with the base station situated in the same cell in which it resides. For future considerations, it will not be necessary to specify the channel access method. We will simply refer to a bidirectional radio link as a single channel. Channel frequencies are permanently assigned to each base station, providing that they are reused in cells that are separated enough to prevent mutual radio interference. This is otherwise known as the Fixed
Channel Allocation (FCA) scheme. With this scheme, greater channel re-use can be achieved by cell size reduction.

In general, users are clustered in classes. Users cannot change their class. Inside their class, users are indistinguishable from each other. A user belonging to a certain class can:

- move from one cell to another, following the mobility parameters which characterize the class,

- make phone calls according to the calling parameters of the class,

- be served by the base station of the cell in which they reside, according to the priority policy assigned to the class.

The mobility parameters are the cell transition rate and the cell transition probability pattern, which can be driven by experimental measures on real systems, such as city traffic measurements. The calling parameters are the phone call rate and the call duration time. This allows us to consider different kinds of users, such as businesses or family ones, in several mobility conditions such as pedestrians or car/jet aircraft passengers.

An attempted phone call is blocked when a base station fails to find a free channel to accommodate a new call. Similarly, a hand-off blocking event occurs when a base station fails to accommodate a user moving into a new cell with a call in progress. In this case the call is forced to terminate.

Typically, we envisage that mission critical calls will require a suitable Quality of Service (QoS), experiencing both low call blocking and low 
forced termination rates. Likewise, high mobility users will be prone to high hand-off rates and, consequently, to high forced termination rates and poor QoS. Conversely, a bunch of low mobility users will be interested only in a cheap service with average QoS. Hence, a well designed mobile communication service should serve users with at least two different priority policies. To this end, users will be clustered in two classes which we will identify as high priority class and low priority class. Users served according to the high priority policy will experience better QoS, lower call blocking and lower forced termination rates than users served according to the low priority. Therefore, considering the aforementioned requirements, high mobility users and mission critical calls are best served in the high priority class. Low mobility users without any special requirements, belonging to the low priority class, still get an average QoS. We will illustrate the model in the Mobility Modeling and the Traffic Modeling sections. An example based on the proposed model will be presented in the Numerical Results section. A discrete event simulator presented in the Simulation Issues section will confirm performance predictions of the model. The Conclusions will close the paper.

\section{Mobility Modeling}

The target of this section is to find user distribution among the cells, considering only their mobility characteristics irrespective of their traffic requests, while in the next section a traffic model will be developed, based on the results of this section. We will assume the radio coverage

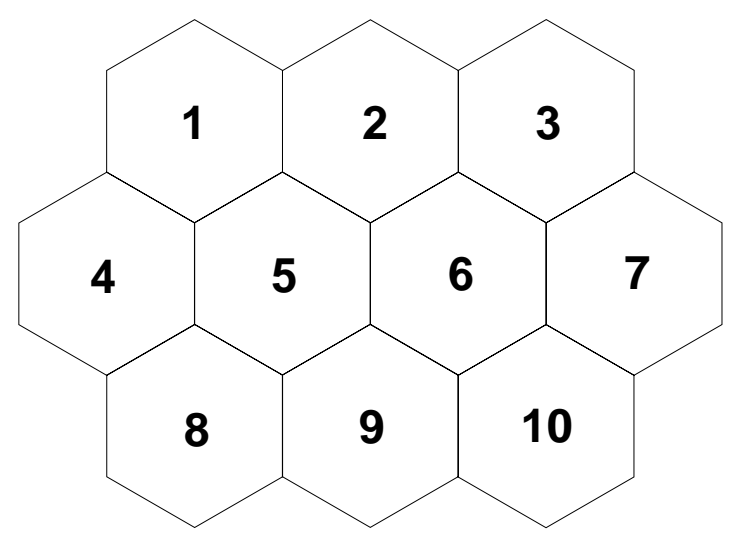

Fig. 1. Cellular Network. of a base station as hexagonal area. Consequently, a cellular network of a finite number of ten cells looks like that in Fig. 1.

As far as the cell dwell time is concerned, each cell is modeled by a queue with infinite servers. This assumption is based on the consideration that a sojourn in a cell is always allowed, regardless of the number of users already present. That is, each user's dwell time in a cell is considered by a virtual server $[1,2]$. The generic transition probability from cell $\mathrm{j}$ to cell $\mathrm{i}$ is indicated by $p_{j ; i}$.

Using this formalization, user mobility is modeled by a closed queueing network which, in the stated hypotheses, admits a product form solution [3]. The following homogeneous system of linear equations provides the arrival rate of class $r$ users at each cell $i\left(\lambda_{i r}\right)(M$ indicates the number of cells)

$$
\lambda_{i r}=\sum_{j=1}^{M} \lambda_{j r} p_{j ; i} \quad i=1,2, \ldots, M
$$

The previous system of equations admits infinite solutions; the values of $\lambda_{i r}$ do not represent the effective arrival rate, but just a relative visit rate to that cell. The true arrival rate will be derived later (5). Inside each generic class $r$ there are $N_{r}$ users. We will assume that in a class $r$ and at cell $j$, the cell dwell time of a generic user is a random variable, $T_{j r}$, with arbitrary p.d.f. and mean $1 / \mu_{j r}$. A state vector $\mathbf{n}=\left(\mathbf{n}_{1}, \ldots, \mathbf{n}_{M}\right)=$ $\left(n_{11}, n_{12}, \ldots, n_{1 R}, \ldots, n_{M 1}, n_{M 2}, \ldots, n_{M R}\right)$ stores the network state, where $n_{i c}$ and $R$ indicate the number of class $c$ users at the cell $i^{\text {th }}$ and the total number of classes. As formerly stated, the dwell time is modeled as the service time of a queue with infinite servers. Applying the BCMP theorem [3], we find the user distribution in the cells $P(\mathbf{n})[4]$ :

$$
P(\mathbf{n})=\frac{1}{G} \prod_{i=1}^{M} \prod_{r=1}^{R} \frac{1}{n_{i r} !}\left(\frac{\lambda_{i r}}{\mu_{i r}}\right)^{n_{i r}} .
$$

The normalization constant $G$, in this specific case, is simply given by (see Appendix):

$$
G=\prod_{r=1}^{R} \frac{1}{N_{r} !}\left(\sum_{i=1}^{M} \frac{\lambda_{i r}}{\mu_{i r}}\right)^{N_{r}} .
$$


The marginal state probabilities with respect to cell $j$ and with respect to cell $j$ and class $r$ are (see Appendix):

$$
\begin{gathered}
p\left(n_{j}\right)=\prod_{r=1}^{R} \mathbf{B}\left(N_{r}, \frac{\frac{\lambda_{j r}}{\mu_{j r}}}{\sum_{i=1}^{M} \frac{\lambda_{i r}}{\mu_{i r}}}\right)\left(n_{j r}\right), \\
p\left(n_{j r}\right)=\mathbf{B}\left(N_{r}, \frac{\frac{\lambda_{j r}}{\mu_{j r}}}{\sum_{i=1}^{M} \frac{\lambda_{i r}}{\mu_{i r}}}\right)\left(n_{j r}\right) .
\end{gathered}
$$

The average number of users of class $r$ in cell $j$ is given by:

$$
\begin{aligned}
\mathbf{E}\left[n_{j r}\right] & =\sum_{k=1}^{N_{r}} k p\left(n_{j r}=k\right) \\
& =\sum_{k=1}^{N_{r}} k \mathbf{B}\left(N_{r}, \frac{\frac{\lambda_{j r}}{\mu_{j r}}}{\sum_{i=1}^{M} \frac{\lambda_{i r}}{\mu_{i r}}}\right)(k) \\
& =N_{r} \frac{\frac{\lambda_{j r}}{\mu_{j r}}}{\sum_{i=1}^{M} \frac{\lambda_{i r}}{\mu_{i r}}}, \quad r=1, \ldots R .
\end{aligned}
$$

Finally, from equation (4) and Little's result, the true arrival rate can be easily evaluated:

$$
\lambda_{j r}\left(N_{r}\right)=\frac{\mathbf{E}\left[n_{j r}\right]}{\mathbf{E}\left[T_{j r}\right]}=\mathbf{E}\left[n_{j r}\right] \mu_{j r}
$$

It is easy to show that, as expected, the throughputs $X_{j r}$, are [2]:

$$
X_{j r}\left(N_{r}\right)=\lambda_{j r}\left(N_{r}\right) .
$$

The finite population mobility model presented in this section is quite general. In fact, the cell dwell time, which can be different from cell to cell and from class to class, can have general distribution and what is actually needed in the model is simply its average value, which can be easily measured experimentally in real systems. Moreover, the generic transition probability from cell $j$ to cell $i, p_{j ; i}$, is completely arbitrary and can also be easily determined by experimental measures in real systems. As an example, in GSM systems, each handover process requires a set of measurements and analyses to choose the target cell. Relevant information is kept in system data bases that allow us to derive an estimation of transition probabilities [5]. A relevant consequence of the model is that user distribution among cells can be nonuniform, capturing, in this way, fairly realistic situations. Globally, computational complexity for deriving system parameters relies on the algorithms efficient for solving the sparse systems of linear equations (1).

\section{Traffic Modeling}

Physical resources of generic cell $j$ are represented by its communication channels $C_{j}$. Base stations administer radio channels according to the channel assignment policy designed for the two classes a user can belong to: high priority or low priority class. This can be achieved if base stations assign channels with priority for high priority users. When a high priority user request (new phone call or hand-off) is denied, the base station forces a low priority user in conversation, if there is one, to terminate his call. The new free channel accommodates the high priority call.

$N_{L}$ users belong to the Low priority class $(L)$, whereas $N_{H}$ users belong to the High priority class $(H)$. Considering a particular network state vector $\mathbf{n}$, we assume that a cell $j^{\text {th }}$ contains $\left(n_{H}, n_{L}\right)$ users. Each idle user generates a new call in an interval of duration $\Delta t$ with the probability $\lambda_{H} \Delta t+o(\Delta t)$ or $\lambda_{L} \Delta t+o(\Delta t)$, depending on the user class; i.e., the time a user remains idle is an exponential random variable with the $\lambda_{H}$ or $\lambda_{L}$ rate, for high and low priority classes, respectively. A suitable description of cell $j$ state is given by the number of channels assigned to high and low priority calls $\left(n_{a_{H}}, n_{a_{L}}\right)$; i.e., the number of users in cell $j$ with a call in progress. We outline the difference between the state of the network and the state of the cell; in other words, the difference between users in a cell (total number of users) and users in the cell with a call in progress (active users).

Poisson driven events are a common assumption among telecom engineers. Call duration 
is modeled by an exponential random variable, $T_{c}$, with mean $1 / \mu_{c}$ which, to keep things simple, is assumed to be the same for high priority and low priority users. The average cell dwell time, assumed to be the same for all cells, will be renamed $1 / \mu_{H}$ and $1 / \mu_{L}$, corresponding to the two exponential random variables $T_{H}$ and $T_{L}$. We assume independence among all these random variables. The previous hypotheses allow us to evaluate the steady state probability $P_{a}\left(n_{a_{H}}, n_{a_{L}}\right)$ in a generic cell $j$ by means of a bidimensional truncated continuous time Markov chain shown in Fig. 2. This can be solved numerically, by standard techniques whose complexity is related to the modest number of channels assigned to each cell.

From these results and observing the states in Fig. 2, we find that a high priority call is blocked only when all channels have been assigned to previous high priority calls of the other $n_{H}-1$ users, i.e.:

$$
P b_{j H}\left(n_{H}, n_{L}\right)=P_{a}(C, 0)
$$

evaluated considering $\left(n_{H}-1, n_{L}\right)$ users in the cell $j[6,7]$. Similarly, low priority calls are blocked when all of the channels have been assigned to previous, either high or low priority calls, i.e.:

$$
P b_{j L}\left(n_{H}, n_{L}\right)=\sum_{i=0}^{C} P_{a}(i, C-i)
$$

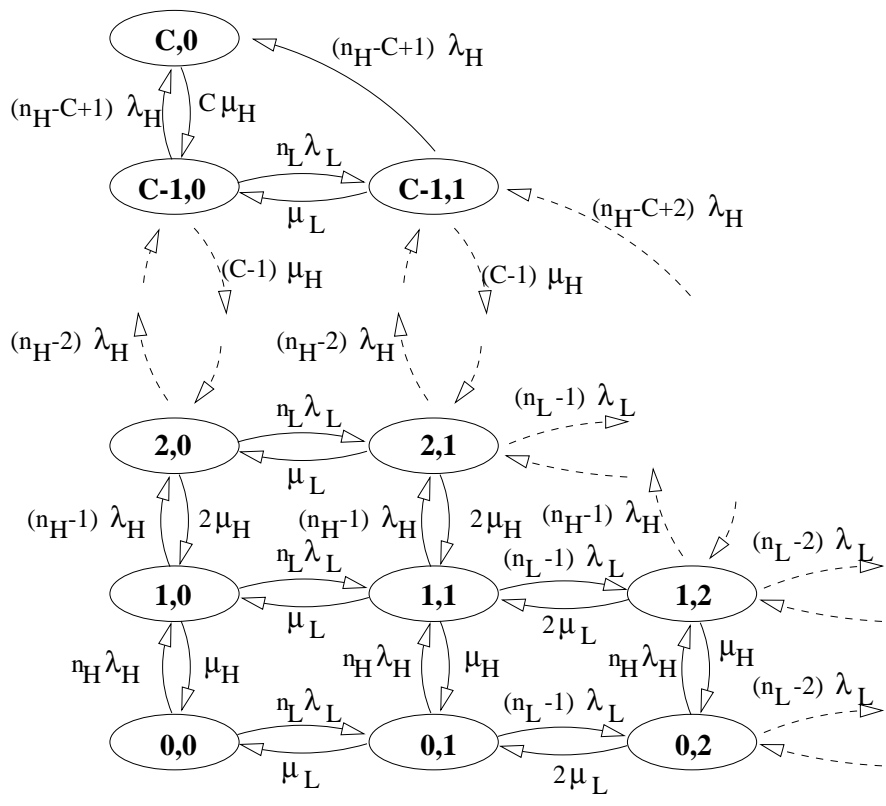

evaluated considering $\left(n_{H}, n_{L}-1\right)$ users in cell $j$. The average number per time unit of low priority calls aborted due to the priority mechanism $\left(N L_{a b t}\right)$, normally terminated $\left(N L_{t r m}\right)$ and accepted $\left(N L_{a c c}\right)$, can easily be worked out by a sum over the appropriate states:

$$
\begin{aligned}
N L_{a b t}= & \sum_{j=1}^{M} \sum_{i=1}^{\min \left(C_{j}, n_{L}^{*}\right)}\left(\left(n_{H}^{*}-\left(C_{j}-i\right)\right) \lambda_{H}+\lambda_{a_{j H}}\right) . \\
& \cdot P_{a}\left(C_{j}-i, i\right), \\
N L_{t r m}= & \sum_{j=1}^{M} \sum_{n_{a_{H}}=0}^{\min \left(C_{j}, n_{H}^{*}\right)} \sum_{n_{a_{L}}=0}^{\min \left(C_{\left.j-n_{a_{H}}, n_{L}^{*}\right)} n_{a_{L}} \mu_{L} \cdot\right.} \\
& \cdot P_{a}\left(n_{a_{H}}, n_{a_{L}}\right), \\
N L_{a c c}= & \sum_{j=1}^{M} \sum_{n_{a_{H}}=0}^{\min \left(C_{j}, n_{H}^{*}\right)} \sum_{n_{a_{L}}=0}^{\min \left(C_{j-n_{a_{H}}-1}, n_{L}^{*}\right)} \\
& \left(\left(n_{L}^{*}-n_{a_{L}}\right) \lambda_{L}+\lambda_{a_{j L}}\right) P_{a}\left(n_{a_{H}}, n_{a_{L}}\right),
\end{aligned}
$$

where $n_{H}^{*}$ and $n_{L}^{*}$ are the closest integers to the average number of high and low priority users in cell $j$ given by (4). Arrival rates of high and low priority active users at cell $j$, respectively $\lambda_{a_{j H}}$ and $\lambda_{a_{j L}}$, will be derived later. Finally, we can derive the probability that a low priority call

Fig. 2. Markov Chain. 
is aborted $\left(P_{a b t}\right)$ or not aborted $\left(P_{n a b}\right)$ by a high priority call:

$$
\begin{aligned}
P_{a b t} & =\frac{N L_{a b t}}{N L_{a c c}} \\
P_{n a b} & =\frac{N L_{a c c}-N L_{a b t}-N L_{t r m}}{N L_{a c c}} \\
& =1-P_{a b t}-\frac{N L_{t r m}}{N L_{a c c}} .
\end{aligned}
$$

\subsection{New Call Blocking}

The probability that a new call attempt is blocked is given by (6) for high priority and (7) for low priority users.

The blocking probability of the entire cellular network is evaluated by means of blocking probabilities of the cells weighted by the fraction of users in the cell; in case of high priority users we have:

$$
P_{B H}(n)=\sum_{j=1}^{M} P b_{j H}\left(n_{j H}, n_{j L}\right) \frac{n_{j H}}{N_{H}} .
$$

Such a quantity is still a function of the network state, so the average blocking probability is given by:

$$
\begin{aligned}
P_{B_{H}}= & \sum_{n_{1 H}} \sum_{n_{1 L}} \sum_{n_{2 H}} \sum_{n_{2 L}} \ldots \sum_{n_{M H}} \sum_{n_{M L}} \\
& \left(\sum_{j=1}^{M} P b_{j H}\left(n_{j H}, n_{j L}\right) \frac{n_{j H}}{N_{H}}\right) . \\
& \cdot P\left(n_{1 H}, n_{1 L}, n_{2 H}, n_{2 L}, \ldots, n_{M H}, n_{M L}\right) \\
= & \sum_{j=1}^{M} \sum_{n_{j H}=C_{j}+1}^{N_{H}} \sum_{n_{j L}=0}^{N_{L}} P b_{j H}\left(n_{j H}, n_{j L}\right) . \\
& \cdot \frac{n_{H}}{N_{H}} P\left(n_{j H}, n_{j L}\right)
\end{aligned}
$$

where $P\left(n_{1 H}, n_{1 L}, n_{2 H}, n_{2 L}, \ldots, n_{M H}, n_{M L}\right)$ and $P\left(n_{j H}, n_{j L}\right)$ represent respectively the users probability distribution in the cells and the probability of having $n_{j H}$ and $n_{j L}$ users in the $j^{\text {th }}$ cell derived by equation (3). The index $n_{j H}$ starts from $\left(C_{j}+1\right)$, because in case of $n_{j H} \leq C_{j}$ (i.e., less high priority users than channels) the blocking probability is obviously zero. Similarly, we can evaluate the low priority users network blocking probability as:

$$
\begin{aligned}
P_{B_{L}}=\sum_{j=1}^{M} & \sum_{n_{j H}=0}^{N_{H}} \sum_{n_{j L}=\max \left(C_{j}+1-n_{j H}, 1\right)}^{N_{L}} \\
& P b_{j L}\left(n_{j H}, n_{j L}\right) \cdot \frac{n_{L}}{N_{L}} P\left(n_{j H}, n_{j L}\right) .
\end{aligned}
$$

Computational complexity for evaluating equations (9) and (10), as can easily be verified, is $O\left(M N_{H} N_{L}\right)$.

\subsection{Hand-off Blocking}

According to the channel assignment policy, the hand-off blocking probability of the $j^{\text {th }}$ cell for both high and low priority users is:

$$
\begin{aligned}
P_{h_{j H}}= & \sum_{n_{j H}=C_{j}+1}^{N_{H}} \sum_{n_{j L}=0}^{N_{L}} P b_{j H}\left(n_{j H}, n_{j L}\right) P\left(n_{j H}, n_{j L}\right), \\
P_{h_{j L}}= & \sum_{n_{j H}=0}^{N_{H}} \sum_{n_{j L}=\max \left(C_{j}+1-n_{j H}, 1\right)}^{N_{L}} P b_{j L}\left(n_{j H}, n_{j L}\right) . \\
& \cdot P\left(n_{j H}, n_{j L}\right) .
\end{aligned}
$$

The departure rate from the $j^{\text {th }}$ cell is estimated as:

$$
\begin{aligned}
& X_{j H}\left(N_{H}\right)= \sum_{n_{j H}=0}^{N_{H}} \sum_{n_{j L}=0}^{N_{L}} \sum_{\substack{n_{a_{H}}=0 \\
n_{a_{H}} \mu_{H} \cdot P_{a}\left(n_{a H}, n_{a L}\right)}}^{\min \left(n_{H}, C_{i}\right)} \sum_{n_{a_{L}}=0}^{\min \left(n_{L}, C_{j}-n_{a_{H}}\right)} \\
&\left.n_{j H}, n_{j L}\right) .
\end{aligned}
$$

Considering the total departure rate of users with a conversation in progress from all the adjacent cells we get:

$$
\lambda_{a_{i H}}\left(N_{H}\right)=\sum_{j=1}^{M} X_{j H}\left(N_{H}\right) p_{j ; i}
$$

The network hand-off blocking probability is a weighted sum of the hand-off probabilities of the cells, where the weight is simply a fraction of the active user arrival rate at the considered cell $\left(\lambda_{a_{j H}}\right)$. For high priority users $(11)$ :

$$
P_{H_{H}}=\sum_{j=1}^{M} P h_{j H} \frac{\lambda_{a_{j H}}\left(N_{H}\right)}{\sum_{i=1}^{M} \lambda_{a_{i H}}\left(N_{H}\right)} .
$$

Similar considerations yield $P_{H_{L}}$ for low priority users. 


\subsection{Forced Termination}

The forced termination probability quantitatively evaluates the quality of service (QoS) offered by the network. The high priority users forced termination probability can be evaluated by taking into account the fact that an active high priority user may switch from one cell to another more than once; the call is aborted when there is a blocked hand-off. Hence we have:

$$
\begin{aligned}
P_{T_{H}}= & \sum_{i=1}^{\infty} P\{\text { Forced Termination } \mid i \text { cells } \\
& \text { switch }\} \cdot p\{i \text { cells switch }\} .
\end{aligned}
$$

Recalling the independence of $T_{c}$ and $T_{H}$ we have:

$$
P(\text { one cell switch })=P\left(T_{c}>T_{H}\right) .
$$

Given the memoryless property of the exponential distribution, we have:

$$
p\{i \text { cells switch }\}=\left[P\left(T_{c}>T_{H}\right)\right]^{i} .
$$

The probability $P\{$ Forced Termination $\mid i$ cells switch $\}$ can be evaluated by considering that the call was blocked at the $i^{t h}$ hand-off and therefore the previous $(i-1)$ hand-off were successful:

$$
\begin{aligned}
P_{T_{H}} & =\sum_{i=1}^{\infty}\left(1-P_{H_{H}}\right)^{i-1} P_{H_{H}}\left[P\left(T_{c}>T_{H}\right)\right]^{i} \\
& =\frac{P_{H_{H}} P\left(T_{c}>T_{H}\right)}{1-\left(1-P_{H_{H}}\right) P\left(T_{c}>T_{H}\right)} .
\end{aligned}
$$

We also have [8]:

$$
\begin{aligned}
P\left(T_{c}>T_{H}\right) & =\int_{0}^{\infty} f_{T_{c}}(t) F_{T_{H}}(t) d t \\
& =\int_{0}^{\infty}\left(1-e^{\mu_{H} t}\right) \mu_{c} e^{-\mu_{c} t} d t \\
& =\frac{\mu_{H}}{\mu_{c}+\mu_{H}} .
\end{aligned}
$$

Similarly, for low priority users we get:

$$
P\left(T_{c}>T_{L}\right)=\frac{\mu_{L}}{\mu_{c}+\mu_{L}} .
$$

Therefore, the forced termination probability is given by:

$$
P_{T_{H}}=\frac{P_{H_{H}} \frac{\mu_{H}}{\mu_{c}+\mu_{H}}}{1-\left(1-P_{H_{H}}\right) \frac{\mu_{L}}{\mu_{c}+\mu_{L}}} .
$$

Yet, for low priority users we split the forced termination into two parts: the first caused by a hand-off failure $\left(P_{T_{L 1}}\right)$, the second by the priority mechanism of high priority calls $\left(P_{T_{L 2}}\right)$.

$$
\begin{aligned}
P_{T_{L 1}}= & \sum_{i=1}^{\infty} P\{\text { Hand-off failure } \mid i \text { cells switch }\} \\
& \cdot p\{i \text { cells switch }\} \\
= & \sum_{i=1}^{\infty}\left(1-P_{H_{L}}\right)^{i-1} P_{H_{L}} P_{n a b}^{i} P\left(T_{c}>T_{L}\right)^{i} .
\end{aligned}
$$

In fact, it has to be considered that the probability of a low priority active user passing from one cell to another includes the probability $P_{n a b}$, where the low priority call has not been aborted by a high priority call in the departure cell. So we have:

$$
\begin{aligned}
P_{T_{L 1}} & =\sum_{i=1}^{\infty}\left(1-P_{H_{L}}\right)^{i-1} P_{H_{L}} P_{n a b}^{i} P\left(T_{c}>T_{L}\right)^{i} \\
& =\frac{P_{n a b} P\left(T_{c}>T_{L}\right) P_{H_{L}}}{1-P_{n a b} P\left(T_{c}>T_{L}\right)\left(1-P_{H_{L}}\right)}
\end{aligned}
$$

The forced termination of high priority users $\left(P_{T_{L 2}}\right)$ due to the priority mechanism splits into more than one term. The first one is related to the situation where a low priority user, remaining in the same cell where the call has begun, is aborted due to the priority mechanism. The second event considers the occurrence that a low priority call is not aborted: it continues until cell transition, then performs a regular handoff, then it is aborted due to a high priority call. Other terms are easily derived:

$$
\begin{aligned}
P_{T_{L 2}} & =P_{a b t}+P_{n a b} P\left(T_{c}>T_{L}\right)\left(1-P_{H_{L}}\right) P_{a b t}+\ldots \\
& =P_{a b t}+\sum_{i=1}^{\infty} P_{a b t} P_{n a b}^{i} P\left(T_{c}>T_{L}\right)^{i}\left(1-P_{H_{L}}\right)^{i} \\
& =P_{a b t} \sum_{i=0}^{\infty}\left(P_{n a b} P\left(T_{c}>T_{L}\right)\left(1-P_{H_{L}}\right)\right)^{i} \\
& =\frac{P_{a b t}}{1-P_{n a b} P\left(T_{c}>T_{L}\right)\left(1-P_{H_{L}}\right)} .
\end{aligned}
$$

Finally, from (21), (22), (19), (13) and (8)

$$
P_{T_{L}}=P_{T_{L 1}}+P_{T_{L 2}} \text {. }
$$




\section{Numerical Results}

Finally, we consider a simple network composed by $M=10$ cells with $N_{H}=50$ high priority users and $N_{L}=50$ low priority users and a common average call duration of $1 / \mu_{c}=180 \mathrm{~s}$. The user mobility is $\mu_{H}=\mu_{L}=20 \mathrm{cells} / \mathrm{h}$. This will result in the same cell switch probability equal to 0.5 . We choose the same transition probability matrix for the two classes (Table 1). Chosen specific values of probability produce, as a result, a non-uniform distribution of users among the cells.
For the sake of simplicity, we will leave out the issue of channel re-use and allocation. We will subdivide available channels into 10 groups, one group for each cell, and we will consider the following non-uniform assignment of 40 channels: $(3,3,3,3,8,8,3,3,3,3)$. Within this framework, we will compare model results (plain lines) and simulation data (symbol ' + '), given by a suitable event-oriented simulation program. Performance results are reported versus user load, defined as the new call generation rate for a single user, which, as explained in section 3 , is given by the reciprocal average idle time. The blocking probability of a new call, for high pri-

\begin{tabular}{c|cccccccccc} 
Cell & 1 & 2 & 3 & 4 & 5 & 6 & 7 & 8 & 9 & 10 \\
\hline 1 & 0 & 0.25 & 0 & 0.25 & 0.5 & 0 & 0 & 0 & 0 & 0 \\
2 & 0.15 & 0 & 0.15 & 0 & 0.35 & 0.35 & 0 & 0 & 0 & 0 \\
3 & 0 & 0.25 & 0 & 0 & 0 & 0.5 & 0.25 & 0 & 0 & 0 \\
4 & 0.25 & 0 & 0 & 0 & 0.5 & 0 & 0 & 0.25 & 0 & 0 \\
5 & 0.15 & 0.15 & 0 & 0.15 & 0 & 0.25 & 0 & 0.15 & 0.15 & 0 \\
6 & 0 & 0.15 & 0.15 & 0 & 0.25 & 0 & 0.15 & 0 & 0.15 & 0.15 \\
7 & 0 & 0 & 0.25 & 0 & 0 & 0.5 & 0 & 0 & 0 & 0.25 \\
8 & 0 & 0 & 0 & 0.25 & 0.5 & 0 & 0 & 0 & 0.25 & 0 \\
9 & 0 & 0 & 0 & 0 & 0.35 & 0.35 & 0 & 0.15 & 0 & 0.15 \\
10 & 0 & 0 & 0 & 0 & 0 & 0.5 & 0.25 & 0 & 0.25 & 0
\end{tabular}

Table 1. Transition probability matrix.

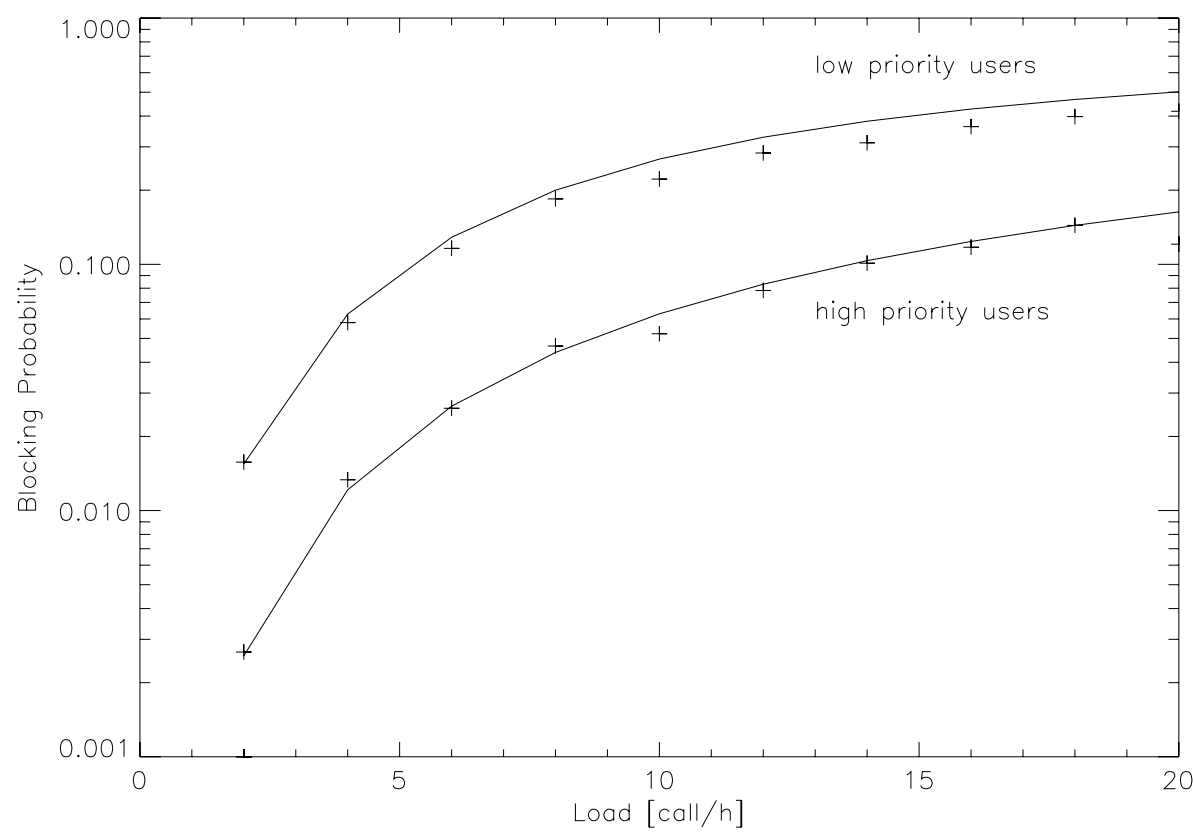

Fig. 3. Blocking Probability vs Load. 


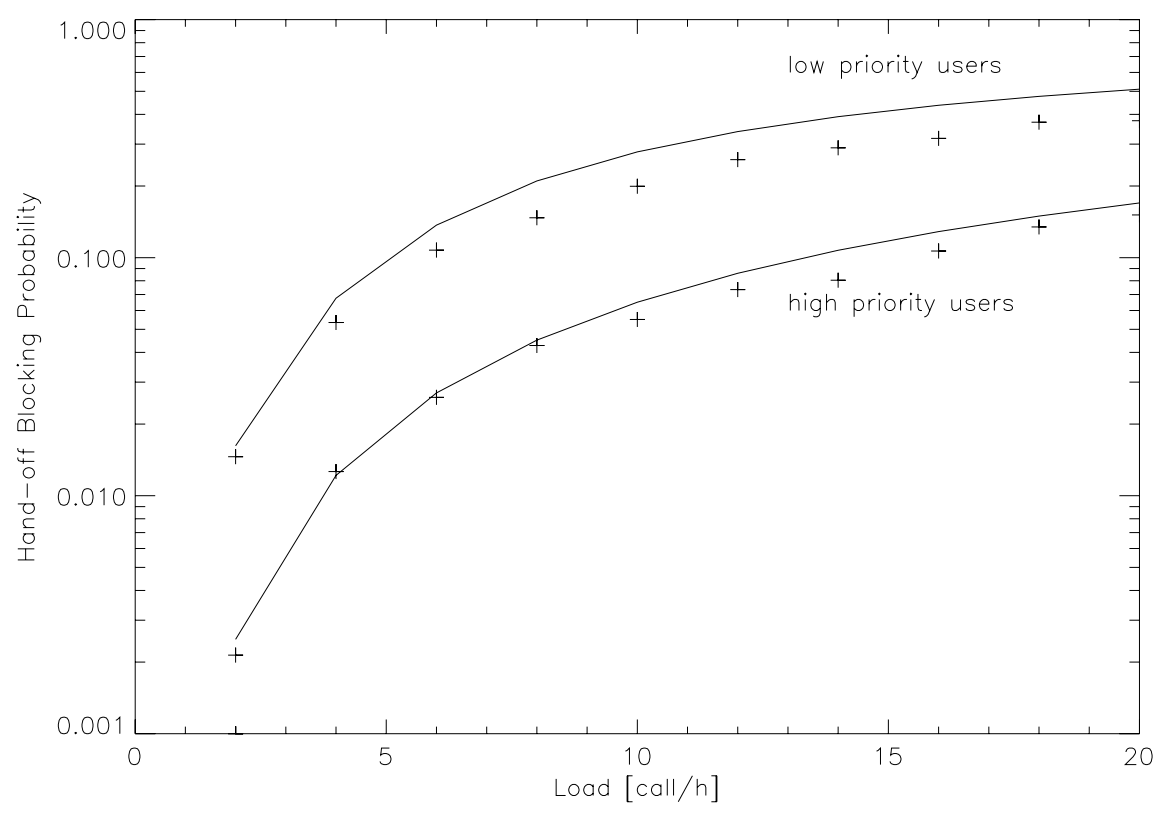

Fig. 4. Hand-off Blocking Probability vs Load.

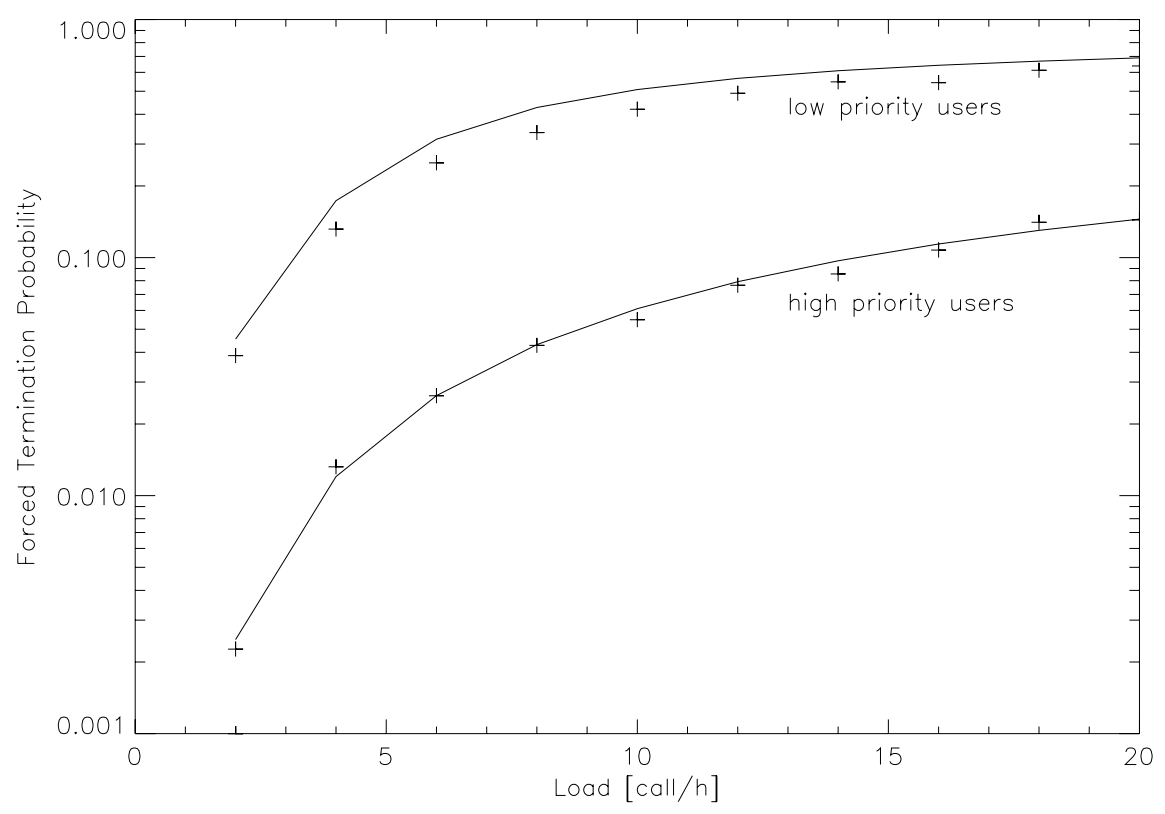

Fig. 5. Forced Termination Probability vs Load.

ority (9) and low priority users (10), is reported versus user load in Fig. 3. As expected, blocking probability rises with the load increase, approaching one as the system collapses. Moreover, an appreciable difference exists between high priority and low priority blocking probability. This difference also exists in the hand-off blocking probability, between high priority (13) and low priority users (Fig. 4). This, of course, is due to the illustrated high priority mechanism. In both cases, model results accurately follow simulation data. A comparison of Fig. 3 with Fig. 4 shows that hand-off blocking probability is quite similar to the blocking probability of a new call. Actually, in each class of users channels are almost equally available for both new call and hand-off attempts. 
Eqs. (9), (10) show that the previously examined probabilities do not depend on mobility. Eq. (13) loosely depends on mobility. Simulation results, not shown, confirm that this is verified from light to moderate load conditions. Fig. 5 shows the forced termination probability of high priority (20) and low priority (23) users versus user load. In this case, results are also confirmed by simulation, especially for light loads.

\section{Simulation Issues}

In order to validate analytical results, a discrete event [15] simulation tool has been implemented. In the system considered, it is not necessary to prevent boundary effects; i.e., simulating a larger system and then collecting data only for the central cells [9]. As a matter of fact, our analytical model includes these effects. In our simulation [10], the system clock advances when one of these events occur: new call start, call termination or cell transition. Cell dwell time, call duration and interarrival times are generated randomly by a suitable Poisson distribution. The cell transition process follows the transition probability matrix. Taking into account both types of users, the number of tried calls $T$, blocked calls $B$, successful calls $S$ and unsuccessful hand-off $H$, we compute new call blocking, hand-off blocking and forced termination probabilities, in each class applying the following:

$$
P_{B}=\frac{B}{T}, \quad P_{H}=\frac{H}{S+H}, \quad P_{T}=\frac{H}{T-B} .
$$

\section{Conclusions}

We have proved that it is possible to model and analyze a multicellular communication network with priority traffic. Hypotheses and approximations of the model are clearly indicated. It is not possible to compare this model with similar analytical models in terms of approach or performance. Indeed, the model developed here is a network model, whereas the models presented in $[9,11,12]$, can be regarded as cell models. Neither of these models groups users into classes.
These models assume an indefinitely large number of undistinguishable mobiles crossing a reference standard cell. This implies that users are assumed to be evenly deployed over the service area and, when referring to the standard cell, not affected by the motion of the mobiles. Network performances are simply a replication of results obtained from this standard cell. Other authors have already found discrepancies regarding the results obtained considering an effectively infinite grouping of cell and results coming from a group of cells $[13,14]$.

The model developed here considers a small group of unevenly loaded isolated cells, which is likely to be the case in a city area. It is based on closed queueing networks [3]. The mobility of the users is modeled by a multi-class infinite servers closed queueing network which admits a product form solution. The channel assignment policy of the cell is modeled by a bi-dimensional truncated continuous time Markov chain whose solution can be found numerically. Considering a finite number of users circulating in the network, it regards each cell with its own number of users (number affected by mobility), its own communication load and its own channel assignment. Moreover, class division allows us to consider two kinds of users in different mobility situations with different quality of service requirements $(\mathrm{QoS})$.

A simple ten cell cellular network has been considered as an example. Model predictions for light to moderate load conditions, cell boundary effects included, have been proven by simulation. As expected, performance decreases at high loads, but the difference in QoS between the two classes of users, in accordance with a likely different billing policy, is confirmed.

As a final consideration, the methodology developed in this paper could be applied to the design of various aspects of cellular networks. As an example, one of these aspects could be the design of Cellular Virtual Private Networks (CVPN), where the number of users is usually quite limited and some services (security, alarm signals, and so on) may require absolute priority. Another example of this application could arise in public cellular networks whose operators have demonstrated an unlimited imagination in proposing new types of contracts. One of these could foresee an economical service with a priority mechanism similar to the one described in this paper. 


\section{Appendix}

The normalization constant $G$ is given by:

$$
\begin{aligned}
& G=\sum P(\mathbf{n})=\sum_{n_{11}, \ldots, n_{M 1}} \ldots \sum_{n_{1 R}, \ldots, n_{M R}} \prod_{i=1}^{M} \frac{\left(\frac{\lambda_{i 1}}{\mu_{i 1}}\right)^{n_{i 1}}}{n_{i 1} !} \ldots \prod_{i=1}^{M} \frac{\left(\frac{\lambda_{i R}}{\mu_{i R}}\right)^{n_{i R}}}{n_{i R} !} \\
& \mathbf{n}, \sum_{i=1}^{M} n_{i r}=N_{r} ; r=1, \ldots, R \quad \sum_{i=1}^{M} n_{i 1}=N_{1} \quad \sum_{i=1}^{n_{11}, \ldots, n_{M 1}} n_{i R}=N_{R} \\
& =\sum_{n_{11}, \ldots, n_{M 1}} \prod_{i=1}^{M} \frac{\left(\frac{\lambda_{i 1}}{\mu_{i 1}}\right)^{n_{i 1}}}{n_{i 1} !} \ldots \sum_{n_{1 R}, \ldots, n_{M R}} \prod_{i=1}^{M} \frac{\left(\frac{\lambda_{i R}}{\mu_{i R}}\right)^{n_{i R}}}{n_{i R} !} \\
& \sum_{i=1}^{M} n_{i 1}=N_{1} \quad \sum_{i=1}^{M} n_{i R}=N_{R} \\
& =\frac{\left(\sum_{i=1}^{M} \frac{\lambda_{i 1}}{\mu_{i 1}}\right)^{N_{1}}}{N_{1} !} \ldots \frac{\left(\sum_{i=1}^{M} \frac{\lambda_{i R}}{\mu_{i R}}\right)^{N_{R}}}{N_{R} !}=\prod_{r=1}^{R} \frac{\left(\sum_{i=1}^{M} \frac{\lambda_{i r}}{\mu_{i r}}\right)^{N_{r}}}{N_{r} !}
\end{aligned}
$$

with the help of the polynomial formula. The arrival rate $\lambda_{i r}$ is evaluated by (1). The marginal state probability with respect to cell $j$ is:

$$
\begin{aligned}
& P\left(\mathbf{n}_{j}\right)=\sum_{\mathbf{n}_{1}, \ldots, \mathbf{n}_{j-1}, \mathbf{n}_{j+1}, \ldots, \mathbf{n}_{M}} P\left(\mathbf{n}_{1}, \mathbf{n}_{2}, \ldots, \mathbf{n}_{M}\right) \\
&=\frac{1}{G} \prod_{r=1}^{R} \frac{\left(\frac{\lambda_{j r}}{\mu_{j r}}\right)^{n_{j r}}}{n_{j r} !} \sum_{\mathbf{n}_{1}, \ldots, \mathbf{n}_{j-1}, \mathbf{n}_{j+1}, \ldots, \mathbf{n}_{M}} \prod_{\substack{i=1 \\
i \neq j}}^{M} \frac{\left(\frac{\lambda_{i 1}}{\mu_{i 1}}\right)^{n_{i 1}}}{n_{i 1} !} \ldots \frac{\left(\frac{\lambda_{i R}}{\mu_{i R}}\right)^{n_{i R}}}{n_{i R} !} \\
&=\prod_{r=1}^{R} \frac{\left(\frac{\lambda_{j R}}{\mu_{j R}}\right)^{n_{j r}}}{n_{j r} !} \sum_{n_{11}, \ldots, n_{j-1}, n_{j+1}, \ldots, n_{M 1}} \prod_{\substack{i=1 \\
i \neq j}}^{M} \frac{\left(\frac{\lambda_{j 1}}{\mu_{i 1}}\right)^{n_{j 1}}}{n_{i 1} !} \frac{N_{1} !}{\left(\sum_{q=1}^{M} \frac{\lambda_{q 1}}{\mu_{q 1}}\right)^{N_{1}}} \ldots \\
& \sum_{n_{1 R}, \ldots, n_{j-1}, n_{j+1}, \ldots, n_{M R}} \prod_{\substack{i=1 \\
i \neq j}}^{M} \frac{\left(\frac{\lambda_{j R}}{\mu_{i R}}\right)^{n_{i R}}}{n_{i R} !} \frac{N_{R} !}{\left(\sum_{q=1}^{M} \frac{\lambda_{q R}}{\mu_{q R}}\right)^{N_{R}}} .
\end{aligned}
$$

Again, applying the polynomial formula we get:

$$
P\left(\mathbf{n}_{j}\right)=\prod_{r=1}^{R} \frac{\left(\frac{\lambda_{j r}}{\mu_{j r}}\right)^{n_{j r}}}{n_{j r} !} \frac{\left(\sum_{\substack{i=1 \\ i \neq j}}^{M} \frac{\lambda_{i 1}}{\mu_{i 1}}\right)^{N_{1}-n_{j 1}}}{\left(\sum_{i=1}^{M} \frac{\lambda_{i 1}}{\mu_{i 1}}\right)^{N_{1}}} \frac{N_{1} !}{\left(N_{1}-n_{j 1}\right) !} \ldots \frac{\left(\sum_{\substack{i=1 \\ i \neq j}}^{M} \frac{\lambda_{i R}}{\mu_{i R}}\right)^{N_{R}-n_{j R}}}{\left(\sum_{i=1}^{M} \frac{\lambda_{i R}}{\mu_{i R}}\right)^{N_{R}}} \frac{N_{R} !}{\left(N_{R}-n_{j R}\right) !}
$$


i.e.,

$$
P\left(\mathbf{n}_{j}\right)=\prod_{r=1}^{R}\left(\begin{array}{c}
N_{r} \\
n_{j r}
\end{array}\right)\left(\frac{\frac{\lambda_{j r}}{\mu_{j r}}}{\sum_{i=1}^{M} \frac{\lambda_{i R}}{\mu_{i R}}}\right)^{n_{j r}}\left(\begin{array}{c}
\sum_{i=1}^{M} \frac{\lambda_{i R}}{\mu_{i R}} \\
\left.\frac{M}{i \neq j}\right)_{i=1}^{M} \frac{\lambda_{i R}}{\mu_{i R}}
\end{array}\right)^{N_{r}-n_{j r}}=\prod_{r=1}^{R} \mathbf{B}\left(N_{r}, \frac{\frac{\lambda_{j r}}{\mu_{j r}}}{\sum_{i=1}^{M} \frac{\lambda_{i R}}{\mu_{i R}}}\right)\left(n_{j r}\right)
$$

where $\mathbf{B}$ is the Binomial distribution. The marginal state probability with respect to cell $j$ and class $r$ is:

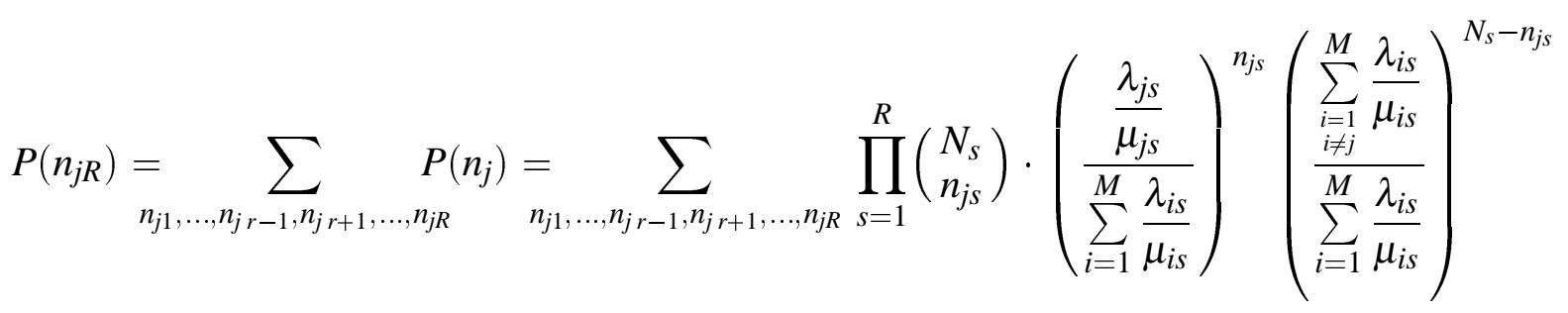

we have

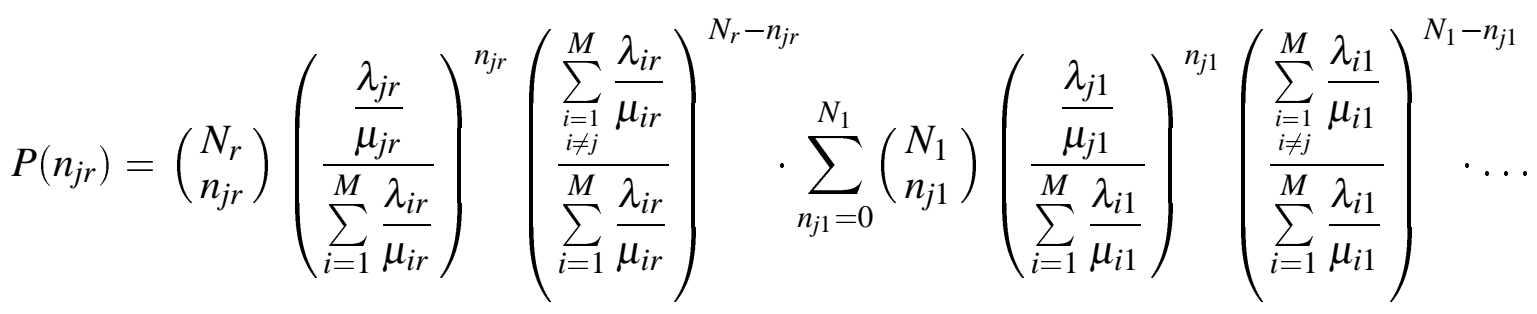

$$
\begin{aligned}
& \ldots \sum_{n_{j R}=0}^{N_{R}}\left(\begin{array}{c}
N_{R} \\
n_{j R}
\end{array}\right)\left(\frac{\frac{\lambda_{j R}}{\mu_{j R}}}{\sum_{i=1}^{M} \frac{\lambda_{i R}}{\mu_{i R}}}\right)^{n_{j R}}\left(\begin{array}{c}
\sum_{\substack{i=1 \\
i \neq j}}^{M} \frac{\lambda_{i R}}{\mu_{i R}} \\
\sum_{i=1}^{M} \frac{\lambda_{i R}}{\mu_{i R}}
\end{array}\right)^{N_{R}-n_{j R}}
\end{aligned}
$$

i.e.,

$$
P\left(n_{j r}\right)=\mathbf{B}\left(N_{r}, \frac{\frac{\lambda_{j r}}{\mu_{j r}}}{\sum_{i=1}^{M} \frac{\lambda_{i r}}{\mu_{i r}}}\right)\left(n_{j r}\right)
$$

recalling the Newton Binomial:

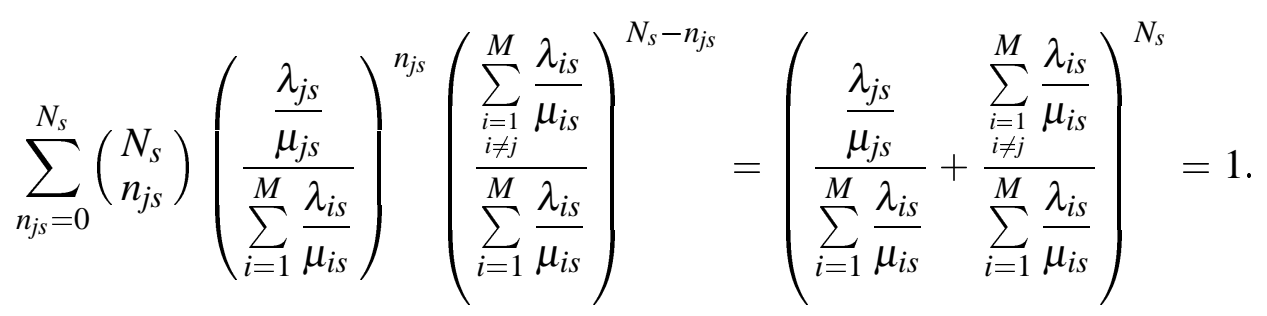


References

[1] P. Camarda, G. Schiraldi, F. Talucci, "Finite Population Model for Performance Evaluation in Cellular Communication Networks", $8^{\text {th }}$ Mediterranean Electrotechnical Conference (MELECON'96), May 96, Bari (Italy).

[2] P. CAMARDA, G. Schiraldi, F. TAlucci, R. VAlla, "Mobility and Performance Modeling in Cellular Communication Networks", ACM Mobile Computing and Communications Review, Vol. 1, N. 4, October 1997.

[3] F. BASKett, K.M. Chandy, R.R. Muntz, F.G. PALACIOS, "Open, closed and mixed networks of queues with different classes of customers", JACM, vol. 22, April 1975, pp. 248-260.

[4] D.L. PAllant, P.G. TAYloR, "Modeling handover in cellular mobile networks with dynamic channel allocation", Operations Research, vol. 43, n. 1, January-February 1995, pp. 33-42.

[5] M. Mouly, M.B. Pautet, “The GSM System for Mobile Communications”, Cell \& Sys, 1992.

[6] M. SCHWARTZ, Telecommunication Networks: Protocols, Modeling and Analysis, Addison Wesley, 1987.

[7] A.L. GARCIA, Probability and Random Processes for Electrical Engineers, Addison Wesley, 1989.

[8] G. SchIRALDI, "Gestione Spettrale in Reti di Comunicazioni Radiomobili”, Dr. Eng. Degree Thesis (in Italian), 1995.

[9] D. HONG, S.S. RAPPAPORT, "Priority Oriented Channel Access for Cellular Systems Serving Vehicular and Portable Radio Telephones", IEE Proceedings, 136(5), Oct. 1989.

[10] M.H. MacDougall, "Simulating Computer Systems: Techniques and Tools", The MIT Press, 1987.

[11] D. Hong, S.S. RAPPAPORT, “Traffic Model and Performance Analysis for Cellular Mobile Radio Telephone Systems with Prioritized and Nonprioritized Handoff Procedures", IEEE Transactions on Vehicular Technology, 35(3), August 1986.

[12] L.H. HU, S.S. RAPPAPORT, "Personal Communication Systems using Multiple Hierarchical Cellular Overlays", IEEE Journal on Selected Areas in Communications, 13(2) February 1995.

[13] D.E. Everitt, N.W. MACFADYEN, "Analysis of Multicellular Mobile Radiotelephone Systems with Loss”, British Telecom Journal, 1(2), October 1983.

[14] M. Sidi, D. STAROBINSKI, "New Call Blocking Versus Handoff Blocking in Cellular Networks", IEEE INFOCOM'96, 1996.

[15] M. H. MACDOUGALL, "Simulating computer systems: techniques and tools", The MIT Press, 1987.
Received: January, 2002

Revised: June, 2003

Accepted: June, 2003

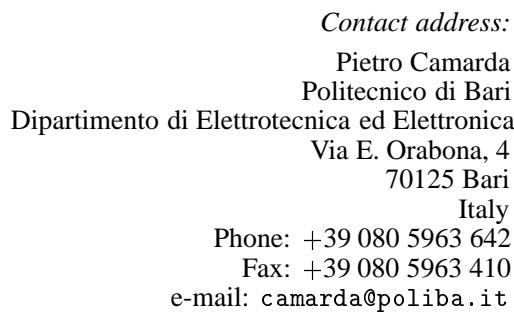

PIETRO CAMARDA received a university degree in computer science from the University of Bari, Italy, in 1978. Since 1986, he has been with the department of "Elettrotecnica ed Elettronica" at the "Politecnico di Bari", Italy, where he is currently an associate professor of telecommunications. From October 1986 to December 1987 he was a visiting scholar at the Computer Science Department of the University of California, Los Angeles (UCLA). He has been involved in various research areas, mainly: LAN/MAN architectures and protocols, optical networks, network reliability, cellular radio networks, telecommunication services, etc.

GIOVANNA SCHIRALDI received the Dr. Eng. degree in electrical engineering from the "Politecnico di Bari", Italy, in 1995. Her research interest include cellular and satellite communication networks.

FABRIZIO TALUCCI received the Dr. Eng. degree in electrical engineering from the "Politecnico di Bari", Italy, in 1993. In 1994 he joined the Italian Navy as an officer. In 1995 he entered the Italian PhD program in electrical engineering at the "Elettrotecnica ed Elettronica" department of the "Politecnico di Bari" and obtained the PhD degree in 1998. He has been a visiting scholar at the Computer Science Department of the University of California, Los Angeles. His research interests span the fields of wireless networking, mobile computing, cellular communication, queueing networking, computer systems and networks performance analysis and evaluation. 